\title{
Eosinophilic pustular folliculitis or Ofuji folliculitis: a rare pathology with a rare complication "renal amyloidosis"
}

\section{Sara Dahhouki, Niema Aqil, Hanane Baybay, Salim Gallouj, Fatima Zahra Mernissi}

Departement of Dermatology, CHU Hassan II, Fès, Morocco

Corresponding author: Dr. Sara Dahhouki, E-mail: dahhoukisara@gmail.com

\begin{abstract}
Eosinophilic pustular folliculitis (EPF) or Ofuji folliculitis is a sterile inflammatory dermatitis of unknown etiology. There are three forms of Ofuji folliculitis: classic Ofuji folliculitis, Ofuji folliculitis associated to immunodeficiency, and Ofuji folliculitis in child. We report a case of a 17-year-old male with a XXY karyotype and congenital insensitivity to pain, who presented follicular and non-follicular papules dispersed in the body with annular disposition. Histology confirmed the Ofuji folliculitis. The evolution of this patient was marked by an oedematous-ascitic syndrome, and the diagnosis of renal amyloidosis was confirmed. Renal amyloidosis is a complication of chronic inflammation, but rarely described in inflammatory skin diseases, and never reported as complication of Ofuji folliculitis.
\end{abstract}

Key words: Ofuji folliculitis; XXY karyotype; Congenital insensitivity to pain; Indomethacin; Renal amyloidosis

How to cite this article: Dahhouki S, Aqil N, Baybay H, Gallouj S, Mernissi FZ. Eosinophilic pustular folliculitis or Ofuji folliculitis: a rare pathology with a rare complication "renal amyloidosis". Our Dermatol Online. 2020;11(e):e44.1-e44.4.

Submission: 23.09.2019; Acceptance: 22.12.2019

DOI: 10.7241 /ourd.2020e.44 


\title{
Folliculite éosinophilique pustulaire ou folliculite d'Ofuji: une pathologie rare avec une complication rare "amylose rénale"
}

\section{Sara Dahhouki, Niema Aqil, Hanane Baybay, Salim Gallouj, Fatima Zahra Mernissi}

Departement of Dermatology, CHU Hassan II, Fès, Morocco

Corresponding author: Dr. Sara Dahhouki, E-mail: dahhoukisara@gmail.com

\begin{abstract}
RÉSUMÉ
La folliculite pustulaire à éosinophilie (EPF) ou folliculite d'Ofuji est une dermatose inflammatoire stérile d'étiologie inconnue. On distingue trois formes de la folliculite d'Ofuji: la folliculite d'Ofuji classique, la folliculite d'Ofuji associée à une immunodéficience par VIH ou sans VIH et la folliculite d'Ofuji associée à l'enfant. Nous rapportons le cas d'un jeune de 17 ans, porteur d'un caryotype XXY, avec une insensibilité congénitale à la douleur, qui présentait des papules folliculaires et non folliculaires en dispositions annulaires dispersés au niveau du corps, don't l'histologie est revenue en faveur de folliculite d'Ofuji. L'évolution de ce malade a été marquee par l'apparition d'un syndrome oedémto-ascitique, don't l'étiologie a été confirmée par une ponction biopsie rénale revenant en faveur d'une amylase rénale. Cette dernière est une complication fréquente d'une inflammation chronique, mais rarement décrite au cours des maladies inflammatoires cutanées, et jamais rapportée comme une complication d'une folliculate d'Ofuji.
\end{abstract}

Mots clé: Folliculite d'Ofuji; Caryotype XYY; Insensibilité congénitale à la douleur; Indométacine; Amylose rénale

\section{INTRODUCTION}

La folliculite pustulaire à éosinophilie (EPF) ou folliculite d'Ofuji est une dermatose inflammation stérile, d'étiologie inconnue, qui a d'abord été décrite initialement par Ise et Ofuji comme une variante de dermatose pustulaire superficielle en 1965 [1]. Cette pathologie présente un pic au cours de la $3^{\text {ème }}$ décennie [2]. Une étude exhaustive sur le travail publiée n×a montré aucune différence de sexe dans l`EPF classique entre 2003 et 2013 [3]. Cette tendance a été confirmée par une enquête épidémiologique nationale menée au Japon au cours de la période de 2010 à 2011 [4,5]. L`éruption de l`EPF se compose de papulo-pustules qui ont tendance à former des plaques annulaires. Histologiquement, 1〉EPF se caractérise par un infiltrat dominé par des éosinophiles dans et autour des unités pilo-sébacées, souvent accompagné d`une formation de micro abcès à éosinophile [6,7]. Ce type d`EPF est actuellement appelé maladie d'Ofuji ou
EPF classique. Nous rapportant un cas de folliculite d'Ofuji dont le diagnostic a été confirmé par l'histologie chez un jeune avec un caryotype XXY.

\section{CASE REPORT}

Un jeune de 17 ans, porteur d'un caryotype XXY, ayant été suivi pour une insensibilité congénitale à la douleur et pour une insuffisance hypothalamo-hypophysaire, et hospitalisé pour prise en charge d'une folliculite d'Ofuji confirmée histologiquement. L'examen clinique décrit des papules folliculaires polygonales de couleur brune, diffuses confluentes en larges placards mal limités, sans avoir tendance à être annulaire, siégeant au niveau des 4 membres, tronc et dos, respectant le visage et le cou (Figs. la - lc). Ces placards sont parsemés par quelques pustules par endroit. Ces lésions étaient asymptomatiques et évoluaient par poussée rémission. La dermoscopie montrait un aspect de papules polygonales, avec éclaircissement

\footnotetext{
How to cite this article: Dahhouki S, Aqil N, Baybay H, Gallouj S, Mernissi FZ. Folliculite éosinophilique pustulaire ou folliculite d'Ofuji: une pathologie rare avec une complication rare "amylose rénale". Our Dermatol Online. 2020;11(e):e44.1-e44.4.

Submission: 23.09.2019; Acceptance: 22.12.2019

DOI: 10.7241 /ourd.2020e.44
} 


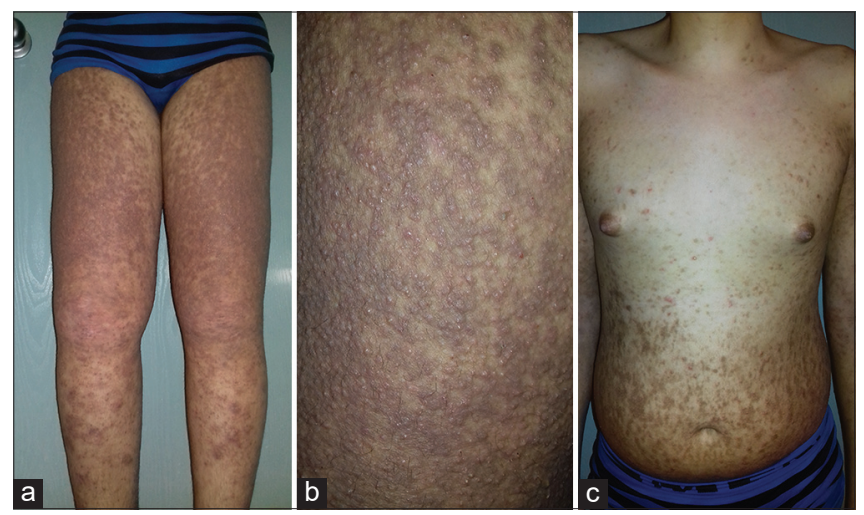

Figura 1: (a-c) Folliculite d'Ofugi; multiples papules cofluants en larges placards.

de leur couleur. La numération formule sanguine ne montrait pas d'hyper éosinophilie. L'histologie objectivait un infiltrat inflammatoire à prédominance de polynucléaires éosinophiles au niveau de la gaine folliculaire, du corps muqueux de Malpighi et du derme papillaire et réticulaire superficiel surtout en périvasculaire (Figs. 2a-2c). Le patient présentait par ailleurs un syndrome oedémato-ascitique d'où la réalisation d'une protéinurie de 24h qui est revenue positive. Une ponction biopsie rénale était réalisée revenant en faveur d'une amylose rénale. Un traitement par indométacine à la dose de $50 \mathrm{mg} / \mathrm{j}$ associé à une préparation magistrale à base de dermocorticoïdes classe très forte avait permis d'obtenir une amélioration satisfaisante. Lévolution de notre patient sous indométacine et des dermocorticoïdes était favorable, avec récidive à l'arrêt. D'où sa mise sous DDS mais l'évolution n'a pas pu être évaluée vu que le patient fut décédé par les complications de son amylose rénale.

\section{DISCUSSION}

La folliculite pustulaire à éosinophilie est une dermatose inflammatoire stérile, prurigineuse, d'étiologie inconnue qui se manifeste cliniquement par des papulo-pustules prurigineuses, qui ont tendance à former des plaques annulaires, évoluant par poussée rémission, surtout au niveau du visage, tronc et membres [8,9], ce qui est le cas chez notre patient. Le prurit manquait chez notre patient vu l'insensibilité congénitale à la douleur. On distingue trois formes de la folliculite d'Ofuji: la folliculite d'Ofuji classique, la folliculite d'Ofuji associée à une immunodéficience par VIH ou sans VIH et la forme de folliculite d'Ofuji associée à l'enfant [9]. La folliculite d'Ofuji n'est pas connue associée à des maladies systémiques [10]. Outre ces trois formes, quelques rares cas de folliculite

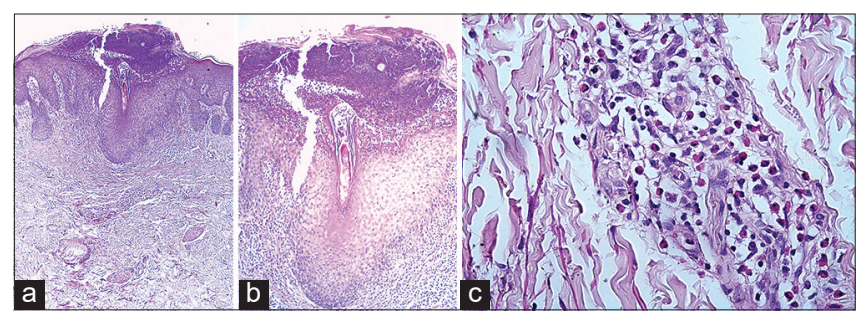

Figura 2: (a-c) Coupes histologiques montrant une pustule folliculaire au niveau infundibulaire, une spongiose de la gaine pilaire et un infiltrat inflammatoire périvasculaire du derme riche en polynucléaires éosinophile.

pustulaire à éosinophiles induite par des médicaments ont également été décrits (allopurinol, carbamazépine ou agents anti-TNF $\alpha$ ) [11-14]. Récemment une folliculite à éosinophilie périodique de la face a été décrite [15]. Histologiquement, l’EPF se caractérise par un infiltrat dominé par des éosinophiles dans et autour des unités pilo-sébacées, souvent accompagné d'une formation de micro abcès à éosinophile $[3,4]$. L'histologie de notre patient montrait un infiltrat inflammatoire à prédominance de polynucléaires éosinophiles au niveau de la gaine folliculaire, du corps muqueux de Malpighi et du derme papillaire et réticulaire superficiel surtout en périvasculaire. Sur le plan thérpeutique, la chronicité et les récidives fréquentes peuvent imposer des traitements souvent lourds, prolongés et parfois multiples [16]. Le traitement de $1^{\text {ère }}$ intention de la folliculite d'Ofuji classique est l'indométacine orale $(25-75 \mathrm{mg} / \mathrm{j})$. En second lieu on peut proposer la photothérapie UVB, la cyclosporine orale $(3-5 \mathrm{mg} / \mathrm{kg} / \mathrm{j})$, les tétracyclines et DDS (50-100mg/j). Le traitement d'entretien, peut être fait, soit par une dégression progressive des médicaments, par l'indométacine topique ou une photothérapie UVB à bande étroite (NB-UVB), 2-3 fois/semaine. On cas de résistance, on peut proposer une thérapie combinée faite d'indométacine ou de DDS avec tacrolimus topique ou indométacine topique ou UVB. Une thérapie combinée à base de tacrolimus et d'UVB doit être évitée [17-21]. Actuellement, aucun traitement n'assure la guérison de l'EPF. Cependant, les lignes de preuve indiquent que la clé est la normalisation de lymphocytes (Th) [22]. Lamylose rénale a été décrit comme une complication de plusieurs pathologies inflammatoire, notamment l'arthrite rhumatoïde, la maladie de Bechterew, rectocolite ulcéro-hémorragique, maladie de Crohn, la spondyloarthrite ankylosante et enfin a été récemment décelée chez $9 \%$ des patients porteurs de la maladie de Still. La pathogénie de cette entité demeure hypothétique. Le traitement de l'amylose rénale secondaire à une inflammation 
chronique est le traitement adéquate de cette dernière. Mais vu que la folliculite pustulaire à éosinophilie est une pathologie récidivante et difficile à traiter, Le traitement de l'amylose rénale suite à la folliculite d'Ofuji sera aussi difficile [23-26].

\section{CONCLUSION}

La folliculite d'Ofuji est une pathologie rare au niveau méditerranéen et africain. Ainsi, l'évolution vers une amylose rénale n'a jamais été décrite. Le cas de notre malade est un cas historique qui révèle une complication grave d'une pathologie chronique difficile à diagnostiquer et à traiter. Nécessitant alors une prise en charge rapide et adéquante.

\section{Consent}

The examination of the patient was conducted according to the Declaration of Helsinki principles.

The authors certify that they have obtained all appropriate patient consent forms. In the form the patient(s) has/have given his/her/their consent for his/her/their images and other clinical information to be reported in the journal. The patients understand that their names and initials will not be published and due efforts will be made to conceal their identity, but anonymity cannot be guaranteed.

\section{REFERENCES}

1. Ise S, Ofuji S. Subcorneal pustular dermatosis A follicular variant. Arch Dermatol. 1965;92:169-71.

2. Razera F, Olm GS, Bonamigo RR. Dermatoses neutrofílicas - Parte II. An Bras Dermatol. 2011;86:195-211.

3. Nomura T, Katoh M, Yamamoto Y, Kabashima K, Miyachi Y. Eosinophilic pustular folliculitis: the transition in sex differences and interracial characteristics between 1965 and 2013. J Dermatol. 2015;42:343-52.

4. Yamamoto Y, Nomura T, Kabashima K, Miyachi Y. Clinical epidemiology of eosinophilic pustular folliculitis: results from a nationwide survey in Japan. Dermatology. 2015;230:87-92.

5. Nervi SJ, Schwartz RA, Dmochowski M. Eosinophilic pustular folliculitis: A 40 year retrospect. J Am Acad Dermatol. 2006;55:285-9.

6. Ofuji S, Ogino A, Horio T, Oseko T, Uehara M. Eosinophilic pustular folliculitis. Acta Derm Venereol. 1970;50:195-203.

7. Ofuji S. Eosinophilic pustular folliculitis. Dermatology. 1987;174:53-6.

8. Anjaneyan G, Manne S, Panicker VV, Eapen M. Ofuji's disease in an immunocompetent patient successfully treated with dapsone. Indian Dermatol Online J. 2016;7:399-401.

9. Dieval C, Denis G, Bolac C, Jazeron J-F. Folliculite à éosinophiles: à propos d'un cas Congrès franc s ais de médecine interne-Deauville, 8 au 10 décembre 2016. Rev Méd Inter. 2016;37:A221.

10. Basarab T, Jones RR. HIV associated eosinophilic folliculitis: Case report and review of the literature. Br J Dermatol. 1996;134:499-503.

11. Mizoguchi S, Setoyama M, Higashi Y, Hozumi H, Kanzaki T. Eosinophilic pustular folliculitis induced by carbamazepine. J Am Acad Dermatol, 1998;38:641-3.

12. Ooi CG, Walker P, Sidhu SK, Gordon LA, Marshman G. Allopurinol induced generalized eosinophilic pustular folliculitis. Australas J Dermatol, 2006;47:270-3.

13. Ramdial PK, Naidoo DK. Drug-induced cutaneous pathology. J Clin Pathol, 2009;62:493-504.

14. Maejima H, Mukai H, Hikaru E. Eosinophilic pustular folliculitis induced by allopurinol and timepidium bromide. Acta Derm Venereol, 2002;82:316-7.

15. Matsumura Y, Miyachi Y. Atypical clinical appearance of eosinophilic pustular folliculitis of seborrheic areas of the face. Eur J Dermatol. 2012;22:658-62.

16. Ziemer M, Böer A. Eosinophilic pustular folliculitis in infancy: not a distinctive inflammatory disease of the skin. Am J Dermatopathol, 2005;27:443-55.

17. Kabashima K, Sakurai T, Miyachi Y. Treatment of eosinophilic pustular folliculitis (Ofuji's disease) with tacrolimus ointment. Br J Dermatol. 2004;151:949-50.

18. Sugita K, Kabashima K, Koga C, Tokura Y. Eosinophilic pustular folliculitis successfully treated with sequential therapy of interferongamma and ciclosporin. Clin Exp Dermatol. 2006;31:709-10.

19. Fukamachi S, Kabashima K, Sugita K, Kobayashi M, Tokura Y. Therapeutic effectiveness of various treatments for eosinophilic pustular folliculitis. Acta Derm Venereol. 2009;89:155-9.

20. Katoh M, Nomura T, Miyachi Y, Kabashima K. Eosinophilic pustular folliculitis: a review of the Japanese published works. J Dermatol. 2013;40:15-20.

21. Nomura T, Katoh M, Yamamoto Y, Miyachi Y, Kabashima K. Eosinophilic pustular folliculitis: A proposal of diagnostic and therapeutic algorithms. J Dermatol. 2016;43:1301-6.

22. Kataoka N, Satoh T, Hirai A, Saeki K, Yokozeki H. Indomethacin inhibits eosinophil migration to prostaglandin D2: therapeutic potential of CRTH2 desensitization for eosinophilic pustular folliculitis. Immunology. 2013;140:78-89.

23. Kilic L, Erden A, Sener YZ, Armagan B, Sari A, Kalyoncu U, et al. Rituximab therapy in renal amyloidosis secondary to rheumatoid arthritis. Biomolecules. 2018;8:136.

24. Stankovic K, Grateau G. Amylose AA. Néphrol Thérap. 2008;4:281-7.

25. Sameh M, Khawla K. Amylose rénale compliquant une maladie de Still de l'adulte: à propos de 3 cas. Pan African Med J. 2019;32:158.

26. Basili E, Cazo M. Amylose compliquant l'évolution d'une rectocolite hémorragique. Gastroentérol Clin Biol. 2002;26:529-31.

Copyright by Sara Dahhouki, et al. This is an open access article distributed under the terms of the Creative Commons Attribution License, which permits unrestricted use, distribution, and reproduction in any medium, provided the original author and source are credited.

Source of Support: Nil, Conflict of Interest: None declared. 